\title{
Ultra-thin optical interference coatings on rough and flexible substrates
}

\section{Citation}

Kats, Mikhail A., and Federico Capasso. 2014. "Ultra-Thin Optical Interference Coatings on Rough and Flexible Substrates." Applied Physics Letters 105 (13): 131108. https:// doi.org/10.1063/1.4896527.

\section{Permanent link}

http://nrs.harvard.edu/urn-3:HUL.InstRepos:41371502

\section{Terms of Use}

This article was downloaded from Harvard University's DASH repository, and is made available under the terms and conditions applicable to Other Posted Material, as set forth at http:// nrs.harvard.edu/urn-3:HUL.InstRepos:dash.current.terms-of-use\#LAA

\section{Share Your Story}

The Harvard community has made this article openly available.

Please share how this access benefits you. Submit a story.

Accessibility 


\title{
Ultra-thin optical interference coatings on rough and flexible substrates
}

\author{
Mikhail A. Kats and Federico Capasso ${ }^{\text {a) }}$ \\ School of Engineering and Applied Sciences, Harvard University, Cambridge, Massachusetts 02138, USA
}

(Received 23 August 2014; accepted 11 September 2014; published online 30 September 2014)

\begin{abstract}
Recently demonstrated ultra-thin optical coatings comprising nanometer-thick highly absorbing films on top of reflecting substrates can display strong optical interference effects, resulting in structural colors and absorption enhancement. Here, we demonstrate that these optical interference effects persist when the films are deposited on substrates that have a large degree of roughness and inhomogeneity on micro- and nano-scales. In particular, we deposited films of gold and amorphous germanium onto paper which serves as a rough and flexible substrate and observe matte interference colors that vary as a function of the germanium thickness. (C) 2014 AIP Publishing LLC.

[http://dx.doi.org/10.1063/1.4896527]
\end{abstract}

Thin film interference is a well-studied and wellunderstood optical effect responsible for the iridescent colors observed in oil films on water and in soap bubbles and is widely used for optical filtering (dichroic, band-pass, neutral density, etc.), anti-reflection and high-reflection coatings, and coloring of materials. ${ }^{1}$ Thin film optical coatings are a mature technology, with many coatings featuring sophisticated multi-layer structures optimized using graphical or computational methods. ${ }^{1,2}$ The vast majority of these structures feature one or more layers of transparent material with thickness approximately equal to or greater than a quarter of the wavelength of light in that material.

Recently, we demonstrated that strong optical interference effects, such as suppression of reflectance and absorption enhancement, can be observed in a simple geometry comprising a highly absorbing continuous film of thickness much smaller than a quarter wavelength of light deposited on top of reflecting substrates. ${ }^{3-5}$ This behavior arises in part as a consequence of the nontrivial (not 0 or $\pi$ ) phase shifts that light experiences upon reflection from an interface where one or both materials have large optical losses (e.g., dielectrics with large imaginary parts $k$ of the complex refractive index $n+i k$ ). Because these structures are simple to fabricate and analyze, they are already finding applications in color coatings, ${ }^{3,6,7}$ solar energy harvesting, ${ }^{8,9}$ anti-reflection coatings, ${ }^{10}$ and static ${ }^{11-14}$ and dynamically tunable ${ }^{4,15}$ absorbers and thermal emitters over wavelengths from the ultraviolet to the far infrared.

One key feature of these ultra-thin optical coatings is that they are significantly less sensitive to the angle of incidence (i.e., less iridescent) compared to conventional thin films because much of the optical phase is accumulated at the interfaces rather than via propagation through the bulk of the film. The strong iridescence observed in conventional thin films is primarily due to optical path length changes in the films for varying viewing angles; different amounts of accumulated optical phase for different angles result in modified interference conditions which determine the observed colors. With only a small portion of phase accumulation occurring via propagation in these ultra-thin films, hardly any iridescence is observed. In fact, it has been

a)capasso@seas.harvard.edu shown that using optical measurements in reflection mode it is impossible to distinguish the ultra-thin-film/substrate structure from a homogeneous semi-infinite block of material with no thin films present at all without prior knowledge about the films as long as the film thickness remains significantly smaller than the wavelength. ${ }^{3,5,16}$ Because of this insensitivity to the angle of incidence, the films can be deposited on substrates that are rough (scattering), such as unpolished silicon wafers, without a significant change in the interference colors. ${ }^{3,7}$ As the films become multilayered and/or increase in overall thickness, the interference colors observed on smooth and rough surfaces begin to differ. ${ }^{7}$ In this work, we demonstrate that ultra-thin interference coatings can tolerate even more dramatically rough substrates and can thus be incorporated onto almost any materials, including flexible ones. We deposit gold $(\mathrm{Au}) /$ germanium $(\mathrm{Ge})$ coatings similar to those in Ref. [3] onto conventional cleanroom paper and observe the same gamut of colors obtained on smooth surfaces with the paper retaining its flexibility.

Using an electron-beam evaporator (Sharon Vacuum), we deposited films of Au and amorphous Ge onto standard cleanroom paper (22 lb, VWR International). The Au films had a thickness of $100 \mathrm{~nm}$ in all samples such that they were approximately opaque and were preceded by a layer of $5 \mathrm{~nm}$ of titanium (Ti). The deposition rates were $1 \mathrm{~A} / \mathrm{s}$ for the $\mathrm{Ti}$ and $2 \mathrm{~A} / \mathrm{s}$ for the $\mathrm{Au}$ and $\mathrm{Ge}$, and the deposition pressure was approximately $2 \times 10^{-6}$ Torr for all films. Cleanroom paper was used instead of conventional paper so as to not contaminate the evaporator. Five samples were made; one with only gold, and the others with $7 \mathrm{~nm}, 10 \mathrm{~nm}, 15 \mathrm{~nm}$, and $100 \mathrm{~nm}$ of $\mathrm{Ge}$, respectively. The thickness was measured using a calibrated crystal monitor.

The resulting samples display a range of interference colors as shown in Fig. 1(a). The top and bottom samples possess the characteristic colors of bulk $\mathrm{Au}$ and amorphous Ge, respectively, whereas the other three samples feature colors which result from the optical interference effect. Because of the scattering characteristics of the underlying paper, all of the samples appear matte. The samples retain the flexibility of paper and do not seem to be affected by repeated bending (Fig. 1(b)). 

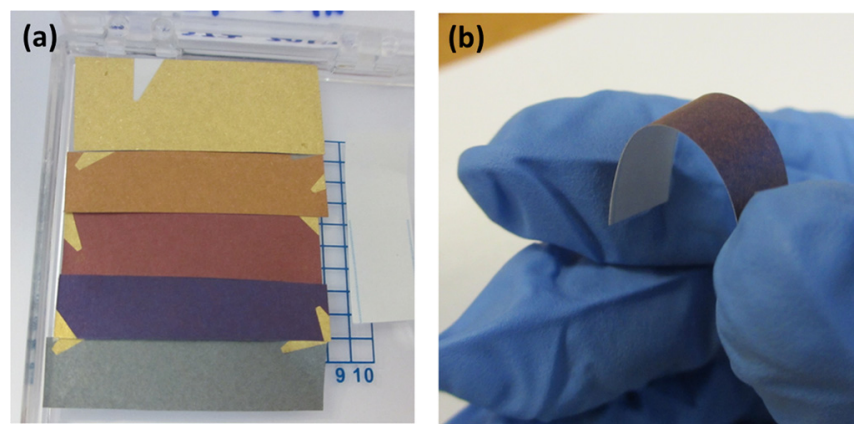

FIG. 1. Photographs of the samples comprising evaporated Au and amorphous Ge on cleanroom paper substrates, taken under illumination from conventional fluorescent ceiling lights. (a) (Top to bottom) optically thick $(100 \mathrm{~nm}) \mathrm{Au}$ on paper with no Ge, and with $7 \mathrm{~nm}, 10 \mathrm{~nm}, 15 \mathrm{~nm}$, and $100 \mathrm{~nm}$ of Ge, respectively. The $100 \mathrm{~nm}$ Ge film is sufficiently thick that the observed color is that of bulk amorphous Ge. (b) A gloved hand bending the sample with $15 \mathrm{~nm}$ of Ge.

To better understand the micro- and nanoscale structure of our paper substrate, we imaged the gold-coated paper with optical profilometry and scanning electron microscopy (SEM). We used a coherence correlation interferometry (CCI) optical profiler (Taylor Hobson), which is able to measure roughness and step-heights with sub-nanometer precision, to image the microscopic structure of our paper (Fig. 2(a)). The 10-15 $\mu \mathrm{m}$ wide fibers comprising the paper are clearly visible. Using an SEM to zoom into a single fiber (Figs. 2(b) and 2(c)), we confirmed that the paper is rough on length scales from hundreds microns to tens of nanometers. Additional SEM images can be found in the supplementary material. ${ }^{20}$

To more rigorously compare the optical response of these optical coatings on paper compared to smooth substrates, we obtained reflection spectra using a visible spectrophotometer (Hitachi 4100, tungsten lamp, photomultiplier tube detector). Because of the matte finish of the films, there is little specular reflection, so we used an integrating sphere to collect the light back-scattered in all directions. The incident light was unpolarized and incident at an angle of approximately $7^{\circ}$ with respect to the sample normal. The resulting spectra are plotted in Fig. 3(a). The reflectivity of

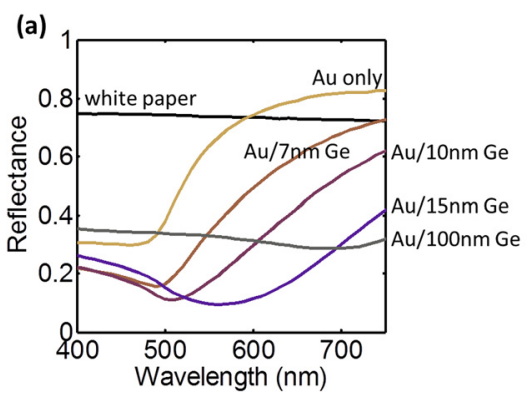

(b) film deposition direction

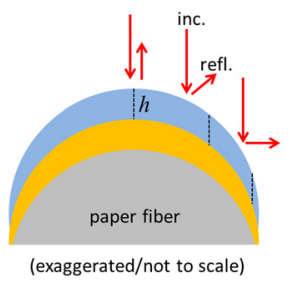

FIG. 3. (a) Near-normal incidence reflectance spectra of the samples in Fig. 1(a) and uncoated white paper. An integrating sphere is used to collect the backscattered light at all angles. (b) Schematic showing the cross-section of a single fiber comprising the paper. The thin films are deposited directionally, which results in a thickness gradient with maximum thickness $\mathrm{h}$ at the top of the fiber and minimum thickness on the sides. Normally incident light samples films of different thicknesses and positioned at different angles.

uncoated paper is high (0.7-0.8) and relatively flat across the visible, as expected for white paper. The Au-only sample has the characteristic reflection spectrum of $\mathrm{Au}$-relatively low at short wavelengths and increasing at longer wavelengths. The film with $100 \mathrm{~nm}$ of Ge has a uniform reflectance of approximately $0.3-0.4$, similar to that of smooth bulk amorphous Ge. The Au-coated samples with $7 \mathrm{~nm}, 10 \mathrm{~nm}$, and $15 \mathrm{~nm}$ Ge films on top feature a dip in reflectance corresponding to a peak in absorbance that is red-shifted across the visible with increasing Ge thickness. This suppression of reflectivity occurs as a result of destructive interference, and the reflectivity curves have the same shape and trend to redshift for increasing thickness observed with $\mathrm{Au} / \mathrm{Ge}$ films on smooth substrates. ${ }^{3}$

The one noticeable difference between the plot in Fig. 3(a) and the corresponding plot in Ref. 3 is that all of the features appear to be blue-shifted when compared to the same film thicknesses on smooth substrates. This indicates that, when deposited on paper, the films are effectively thinner compared to their flat counterparts. There are two reasons for this behavior. The first reason has to do with the directional nature of thin film evaporation: because the evaporated material impinges uniformly on the approximately cylindrical paper fibers, the film thickness will vary (a)

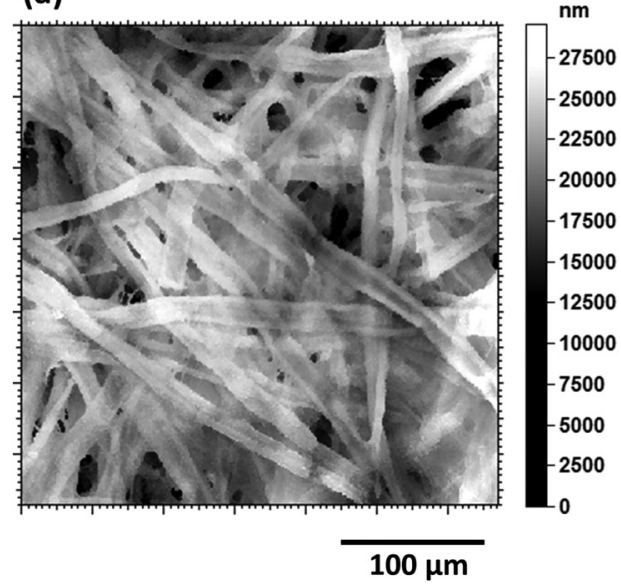

(b)

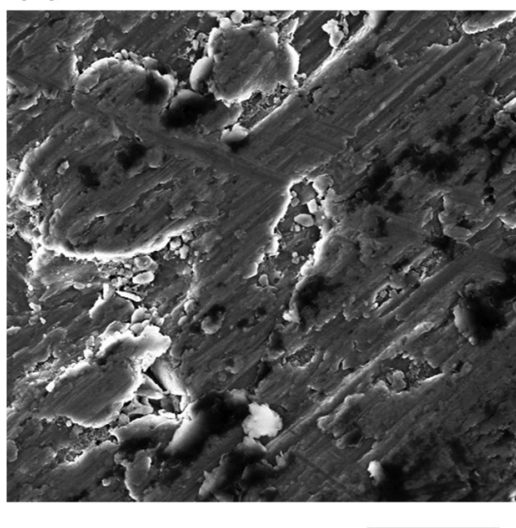

$4 \mu \mathrm{m}$ (c)

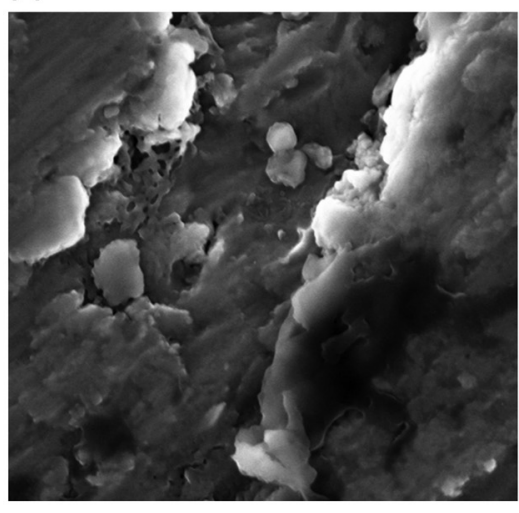

$800 \mathrm{~nm}$

FIG. 2. Images of the paper substrate after deposition of the $100 \mathrm{~nm}$ Au film. (a) Optical profiler image showing the paper fibers. The color bar represents the relative height. (b) and (c) SEM images zooming into a single paper fiber. 
between the thickness read by the crystal monitor at the "horizontal" part of the fiber to no film at all on the "vertical" side walls (Fig. 3(b)). As a result, light hitting different parts of the fiber appears to sample different film thicknesses which are on average smaller than the thickness measured by the crystal monitor. This film non-uniformity also leads to color variation for different angles on our sample which can be seen in Fig. 1(b); while the thin films themselves have minimal iridescence as discussed above, the films are actually thinner on the sides than on the top which leads to a different color when the sample is observed at a large-enough angles of incidence. The non-uniformity can be removed by using a less directional and hence more conformal deposition technique such as sputtering or evaporation with substrate rotation and tilt. The second reason for the blue-shift of the reflectivity dip is intrinsic to any rough surface: by definition, any rough surface will have interfaces at different angles with respect to the incident light (Fig. 3(b)) which affects the reflection spectrum. For ultra-thin Ge films on $\mathrm{Au}$, the minimum in reflectivity occurs at roughly the same wavelength for increasing angle of incidence in the case of s-polarized light, but blue-shifts for p-polarized light. ${ }^{3}$ Since we use unpolarized light in our experiments which is roughly $50 \%$ s-polarized and $50 \%$ p-polarized, the overall reflectivity minimum is expected to blue-shift slightly, which is indeed what we observe in our measurements (Fig. 3(a)).

We note that utilizing a rough disordered substrate for interference coatings is conceptually similar to the use of disordered flakes of optical thin films in pigments. ${ }^{17-19}$ Optical interference effects in ultra-thin films, such as those demonstrated here, may be used to create flake-based pigments while using much less thin film material.

In summary, we have demonstrated that thin film optical interference effects based on ultra-thin highly absorbing films persist on substrates with a large degree of roughness and inhomogeneity. We deposited gold/germanium coatings onto paper and showed that the colored paper remains flexible and features roughly the same interference colors when compared to the same coatings deposited on planar substrates. We posit that almost any substrate can be used in a similar fashion including flexible and compliant materials such as cloth, foam, and rubber. These ultra-thin films can be used to color a variety of substrates using a minimal amount of material and may become inexpensive and easy-to-fabricate light-gathering components for flexible optoelectronic devices.

We acknowledge helpful discussions with David Clarke, Romain Blanchard, Yu Yao, Patrice Genevet, Francesco Aieta, and Jennifer Choy. We also thank Eric Mazur for access to his spectrophotometer and Yu-Ting Lin for assistance with the instrument. The thin film deposition was performed at the Harvard Center for Nanoscale Systems (CNS), which is a member of the National Nanotechnology Infrastructure Network (NNIN). We acknowledge financial support from the Air Force Office of Scientific Research (AFOSR) under Grant No. FA9550-12-1-0289 and from Draper Laboratory.

${ }^{1}$ H. A. Macleod, Thin-Film Optical Filters (Adam Hilger Ltd, Bristol, 1986).

${ }^{2}$ Examples of commercial software for thin film calculations: Essential Macleod, FilmStar, Film Wizard, and OptiLayer.

${ }^{3}$ M. A. Kats, R. Blanchard, P. Genevet, and F. Capasso, "Nanometer optical coatings based on strong interference effects in highly absorbing media," Nat. Mater. 12, 20 (2013).

${ }^{4}$ M. A. Kats, D. Sharma, J. Lin, P. Genevet, R. Blanchard, Z. Yang, M. M. Qazilbash, D. N. Basov, S. Ramanathan, and F. Capasso, "Ultra-thin perfect absorber employing a tunable phase change material," Appl. Phys. Lett. 101, 221101 (2012).

${ }^{5}$ M. A. Kats, R. Blanchard, S. Ramanathan, and F. Capasso, "Thin-film interference in lossy, ultra-thin layers," Opt. Photonics News 25, 40 (2014).

${ }^{6}$ M. A. Kats, S. J. Byrnes, R. Blanchard, M. Kolle, P. Genevet, J. Aizenberg, and F. Capasso, "Enhancement of absorption and color contrast in ultra-thin highly absorbing optical coatings," Appl. Phys. Lett. 103, 101104 (2013).

${ }^{7}$ F. F. Schlich and R. Spolenak, Appl. Phys. Lett. 103, 213112 (2013).

${ }^{8}$ H. Dotan, O. Kfir, E. Sharlin, O. Blanck, M. Gross, I. Dumchin, G. Ankonina, and A. Rothschild, "Resonant light trapping in ultrathin films for water splitting," Nat. Mater. 12, 158-164 (2013).

${ }^{9}$ J. Y. Lee, K.-T. Lee, S. Seo, and L. J. Guo, "Decorative power generating panels creating angle insensitive transmissive colors," Sci. Rep. 4, 4192 (2014).

${ }^{10}$ S. P. Huber, R. W. E. van de Kruijs, A. E. Yakshin, E. Zoethout, K. J. Boller, and F. Bijkerk, "Subwavelength single layer absorption resonance antireflection coatings," Opt. Express 22, 490 (2014).

${ }^{11}$ W. Streyer, S. Law, G. Rooney, T. Jacobs, and D. Wasserman, "Strong absorption and selective emission from engineered metals with dielectric coatings," Opt. Express 21, 9113 (2013).

${ }^{12}$ K. Liu, B. Zeng, H. Song, Q. Gan, F. J. Bartoli, and Z. H. Kafafi, "Super absorption of ultra-thin organic photovoltaic films," Opt. Commun. 314, 48 (2014).

${ }^{13}$ H. Song, L. Guo, Z. Liu, K. Liu, X. Zeng, D. Ji, N. Zhang, H. Hu, S. Jiang, and Q. Gan, "Nanocavity enhancement for ultra-thin optical absorber," Adv. Mater. 26, 2737 (2014).

${ }^{14}$ J. W. Cleary, R. Soref, and J. R. Hendrickson, "Long-wave infrared tunable thin film perfect absorber utilizing highly doped silicon-on-sapphire," Opt. Express 21, 19363 (2013).

${ }^{15}$ M. A. Kats, R. Blanchard, S. Zhang, P. Genevet, C. Ko, S. Ramanathan, and F. Capasso, "Vanadium dioxide as a natural disordered metamaterial: perfect thermal emission and large broadband negative differential thermal emittance," Phys. Rev. X 3, 041004 (2013).

${ }^{16}$ See the supplementary information of Ref. 3, available online at www.nature.com/nmat/.

${ }^{17}$ G. Pfaff and P. Reynders, "Angle-dependent optical effects deriving from submicron structures of films and pigments," Chem. Rev. 99, 1963 (1999).

${ }^{18}$ T. A. Germer and M. E. Nadal, "Modeling the appearance of special effect pigment coatings," Proc. SPIE 4447, 77 (2001).

${ }^{19}$ G. B. Smith, A. Gentle, P. Swift, A. Earp, and N. Mronga, "Coloured paints based on coated flakes of metal as the pigment, for enhanced solar reflectance and cooler interiors: description and theory," Sol. Energy Mater. Sol. Cells 79, 163 (2003).

${ }^{20}$ See supplementary material at http://dx.doi.org/10.1063/1.4896527 for additional SEM images. 